\title{
Membrane Separator Interface for Mass-Spectrometric Analysis of Desflurane, Propofol and Fentanyl in Plasma and Cerebrospinal Fluid
}

\author{
V. Yu. Cherebillo1, V. N. Sokolov², V. A. Elokhin'2, V. I. Nikolaev², A. V. Polegaev, \\ A. Yu. Elizarov ${ }^{*}$ \\ ${ }^{1}$ Kirov Medical Academy, St. Petersburg, Russia \\ ${ }^{2}$ Scientific Instruments CJSC, St. Petersburg, Russia \\ ${ }^{3}$ Ioffe Physical-Technical Institute, St. Petersburg, Russia \\ Email: *a.elizarov@mail.ioffe.ru
}

Received 2 July 2015; accepted 26 October 2015; published 29 October 2015

Copyright (C) 2015 by authors and Scientific Research Publishing Inc.

This work is licensed under the Creative Commons Attribution International License (CC BY).

http://creativecommons.org/licenses/by/4.0/

(c) (i) Open Access

\begin{abstract}
Mass-spectrometric interface for the measurement of anaesthetic agent concentration in biological fluids (blood plasma and cerebrospinal fluid) is described. Sampling of biological fluids was performed during balanced inhalational (desflurane, fentanyl) anaesthesia and total intravenous (propofol, fentanyl) anaesthesia. The described method for drug concentration measurement in biologic fluids does not require long-term sample processing before injecting the sample into massspectrometer interface, in contrast to chromatographic methods. A hydrophobic membrane was used in the interface to separate anaesthetic agents from biological fluids: inhalational anaesthetic desflurane, hypnotic propofol, analgesic fentanyl. A possibility to use the interface for measurement of desflurane and propofol absolute concentration in blood plasma and cerebrospinal fluid was demonstrated for the study of blood-brain barrier (BBB) properties.
\end{abstract}

\section{Keywords}

Membrane, Mass-Spectrometer, Anesthesia, Desflurane, Fentanyl

\section{Introduction}

Chromatographic-mass-spectrometric methods for the analysis of biological fluids require long-term sample

\footnotetext{
*Corresponding author.
}

How to cite this paper: Cherebillo, V.Yu., et al. (2015) Membrane Separator Interface for Mass-Spectrometric Analysis of Desflurane, Propofol and Fentanyl in Plasma and Cerebrospinal Fluid. International Journal of Medical Physics, Clinical Engineering and Radiation Oncology, 4, 263-267. http://dx.doi.org/10.4236/ijmpcero.2015.44031 
processing which is associated with single extraction procedure or head space-gas chromatography with solid phase microextraction last time. Existing methods to assay small molecules in human whole blood, brain, liver, and adipose tissue include high-performance liquid chromatographic determination using direct UV fluorimetric or mass detection [1] [2]. Second-derivative UV spectroscopy and gas chromatography have also been used to assay small molecules [3]. To address practical problems of anaesthesiology (including monitoring of anaesthesia depth and assessment of blood-brain barrier permeability), development of an express method is relevant to allow measuring concentration of anaesthetics in biological fluids in clinical practice, i.e. without long-term multi-stage sample processing.

Currently, methods exist for absolute concentration measurement of wide spectrum of organic compounds dissolved in water. These methods demonstrate high analytical potential of mass-spectrometer membrane interface. Thus, using quadrupole mass-spectrometer, a limit of detection for drug concentration as good as $10^{-8}$ $10^{-7},\left(0.1^{-5} \mu \mathrm{g} / \mathrm{L}\right)$ was achieved [4].

Disadvantages of membrane interfaces include longer response time compared to capillary injection directly into the ion source of mass-spectrometer, dependence of membrane characteristics on the temperature, and selectivity (transmission coefficient) for various compounds.

\section{Material and Methods}

All clinical studies were approved by the administration of the Kirov Medical Academy.

All patients gave written consent to participate in the investigation.

\subsection{The Mass Spectrometry System}

In the present work, a mass-spectrometric membrane separator interface with a $75 \mu$ m polydimethylsiloxane membrane was used for the first time to investigate concentration of anaesthetic agents in biological fluids. A titanium membrane was used to fix the membrane on the aperture of the interface flange $10.0 \mathrm{~mm}$ in diameter. A system of differential pumping provided a pressure drop of 1000 mbar-3.0 $\times 10^{-2}$ mbar-4.0 $\times 10^{-6}$ mbar, where $3.0 \times 10^{-2}$ mbar presented pressure in the differential chamber, $4.0 \times 10^{-6}$ mbar-pressure in the mass-spectrometer chamber, respectively. Interface design allowed heating it to $45 \mathrm{C}$.

The mass-spectrometer chamber and the differential pumping chamber were vacuumed using a turbomolecular pump and the first (molecular) pumping stage of the same pump. Pumping rate for the mass-spectrometer chamber and the differential chamber was $60 \mathrm{~L} / \mathrm{s}$ and $20 \mathrm{~L} / \mathrm{s}$, respectively. These chambers are separated by a diaphragm $100 \mu \mathrm{m}$ in diameter (Pfeiffer Vacuum). A quadruple mass-spectrometer (PrismaPlus, Pfeiffer Vacuum) was used in the study.

\subsection{Anesthesia}

Before induction of anaesthesia, all patients were pre-oxygenated for $1.0 \mathrm{~min}$. In the TIVA group anaesthesia was induced with propofol (Fresenius) $1.0 \mathrm{mg} \cdot \mathrm{kg}^{-1}$ and fentanyl $5.0 \mu \mathrm{g} \cdot \mathrm{kg}^{-1}$. One min after starting anesthesia, pipecuronium bromide $0.6 \mathrm{mg} \cdot \mathrm{kg}^{-1}$ was administered in both groups to achieve muscle relaxation. After tracheal intubation, all patients were ventilated mechanically by using a constant fresh gas $0.5 \mathrm{~L} \cdot \mathrm{min}^{-1}$.

Anesthesia was maintained by a continuous infusion of propofol $3.0 \mathrm{mg} \cdot \mathrm{kg}^{-1} \cdot \mathrm{h}^{-1}$ and fentanyl $0.1 \mathrm{mg}$ over a 20 min period in the TIVA group and with desflurane $6.0 \pm 0.5$ vol\% minimum alveolar concentration (MAC). At the end of surgery, anaesthesia was stopped and the patients' lungs were ventilated with fresh gas $7.0 \mathrm{~L} \cdot \mathrm{min}^{-1}$. The balanced inhalational anesthesia was performed with the inhalational anesthetic agent desflurane (Baxter Healthcare) in a dosage of MAC.

\section{Results and Discussion}

Figure 1 shows a section of a mass-spectrum for gas mixture dissolved in blood plasma, obtained using the membrane interface. Mass peaks of $51 \mathrm{~m} / \mathrm{z}, 101 \mathrm{~m} / \mathrm{z}, 149 \mathrm{~m} / \mathrm{z}$ correspond to desflurane $\left(\mathrm{C}_{3} \mathrm{H}_{2} \mathrm{~F}_{6} \mathrm{O}\right) . \mathrm{A}_{10.0 \mu \mathrm{l}}$ sample was injected directly onto the interface membrane into a closed chamber located immediately above the membrane. Blood and cerebrospinal fluid samples were pre-centrifuged for $10 \mathrm{~min}$ at a $5000 \mathrm{rpm}$ rate. Sampling was performed in vivo during balanced inhalational anaesthesia with desflurane at a concentration of $6.0 \%$ vol in the breathing circuit (BC) of the inhalational anaesthesia machine (IAM). Blood samples were drawn from 


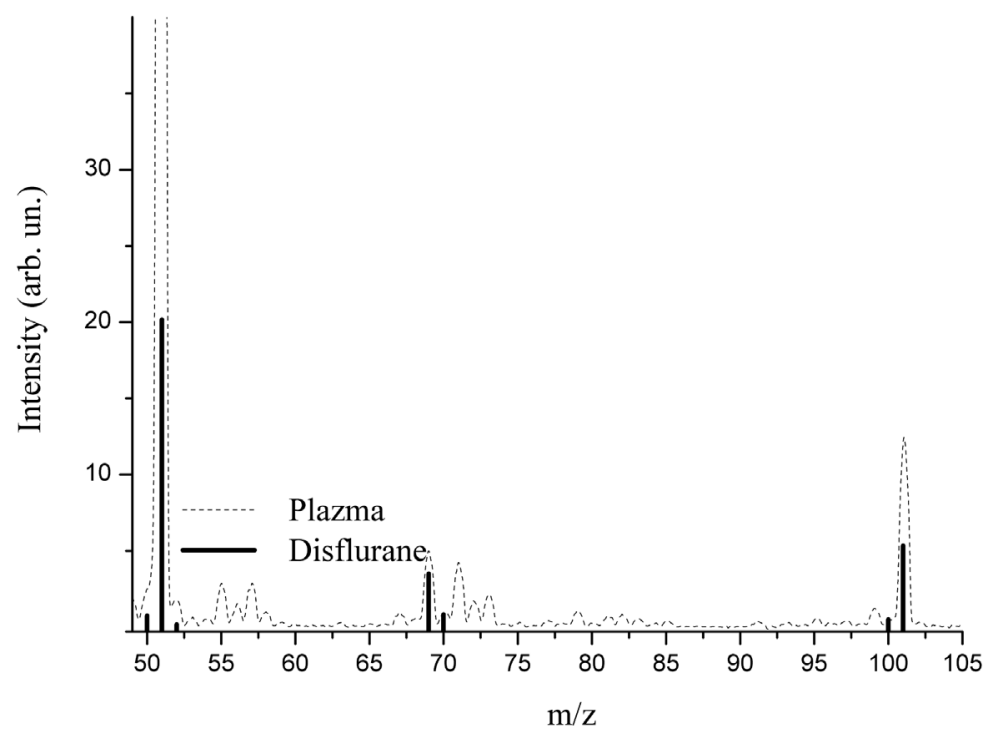

Figure 1. Mass-spectrum of desflurane and desflurane in the plasma that was taken during balanced inhalational anaesthesia, obtained using membrane separator interface.

peripheral vein (PV) and from surgical wound in the chiasmo-sellar area of the brain (CSAB) during adenomectomy of pituitary tumor. The ratio of desflurane concentration in PV (Plasma (PV)) and in CSAB (Plasma $(\mathrm{PV}) /$ Plasma(CSAB)) was $5.4 \pm 1.4$. The indicated difference in concentration is due to the properties of the BBB [5]. BBB lies between endothelial cells lining blood capillaries in the brain; therefore, the anaesthetic agent concentration in the blood from surgical wound in CSAB represents its concentration after passing through BBB. The ratio of desflurane concentrations in PV and in cerebrospinal fluid (CSF)—plasma (PV)/CSF $=1.25 \pm 0.2$ (see Figure 2). Sampling of cerebrospinal fluid was performed during ventriculoperitoneal shunting (VPS) associated with the necessity to compensate increased intracranial pressure. Because of increased intracranial pressure, CSF is accumulated in intercellular space, which leads to vasogenic brain edema causing an increase in BBB permeability. The difference between Plasma (PV)/Plasma (CSAB) and Plasma (PV)/CSF is mainly due to the fact that pituitary adenoma (when Plasma (PV) and Plasma (CSAB) samples were taken) does not lead to increase in BBB permeability, in contrast to CSF sampling during VPS for intracranial pressure compensation. The results were obtained during 12 anaesthesia procedures. It should be noted that a BBB properties study, i.e. assessment of drug ratio in brain tissue and in blood is $\operatorname{logBB}=$ Brain/Blood, where Brain is drug concentration in brain tissue and Blood is blood concentration, respectively, requires expensive and long-term studies since brain tissue samples are taken from the patients who died during the surgery [6] [7]. These results are used in pharmacology and for developing a mathematical model for analytical calculations of $\operatorname{logBB}$ [8]. To find the proportionality factor for $\operatorname{logBB}$ and Plasma (PV)/Plasma (CSAB), additional studies are required to increase statistical significance of the obtained results.

Throughout the anaesthesia opioid analgesic fentanyl (State Plant for Drugs Manufacturing) $\left(\mathrm{C}_{22} \mathrm{H}_{28} \mathrm{~N}_{2} \mathrm{O}\right) 0.1$ $\mu \mathrm{g} / \mathrm{mL}$ was administered every 20 minutes. The fentanyl mass-spectrum in the plasma is shown in the Figure 3. Estimation of the ratio of fentanyl concentration in PV and CSAB was performed in the present study. Plas$\mathrm{ma}(\mathrm{PV}) /$ Plasma $(\mathrm{CSAB})=1.0 \pm 0.2$ (see Figure 2) and, therefore fentanyl enters the central nervous system (CNS) without obstruction, which is consistent with the result obtained in rats [9].

During total intravenous anaesthesia, infusion regimen was used controlled by the target propofol $\left(\mathrm{C}_{12} \mathrm{H}_{18} \mathrm{O}\right)$ concentration in blood. A concentration of $2 \mu \mathrm{g} / \mathrm{mL}$ was selected and maintained by a syringe pump (B|Braun) under control of the infusion regimen controlled by target concentration program-“Stanpump” [10]. The massspectrum of blood samples taken during the total intravenous anaesthesia with propofol and fentanyl is shown in the Figure 4. Intravenous hypnotic propofol is practically insoluble in water, but is highly soluble in lipids, therefore this drug is administered intravenously as an emulsion that includes: $10 \%$ soya bean oil, $1.2 \%$ purified egg phospholipids (emulsifier), 2.25\% glycerol, water, and sodium hydroxide. Emulsified propofol formulation was marketed in 1986 and currently is a hypnotic of choice for inhalational or intravenous anaesthesia. The 


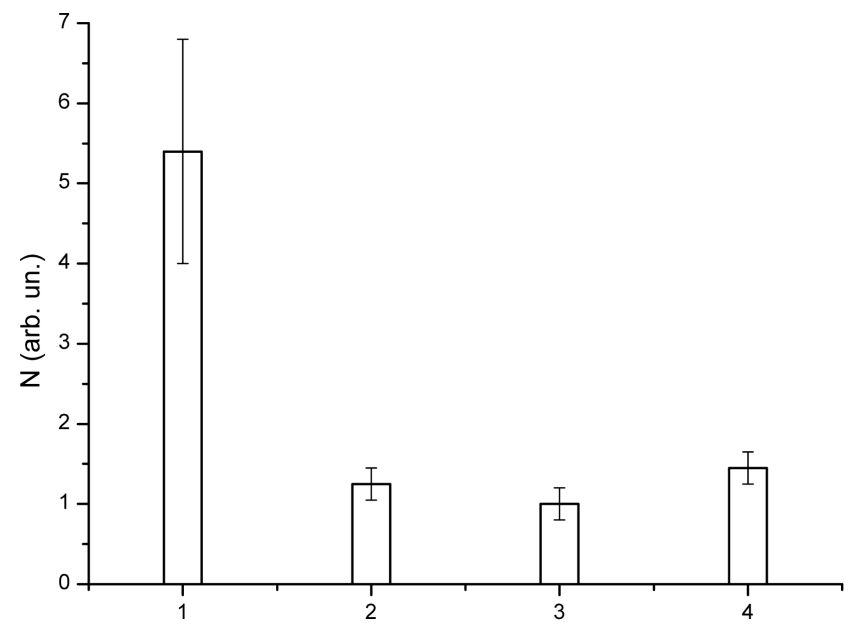

Figure 2. Ratio of drug concentrations in a peripheral vein and in the brain.

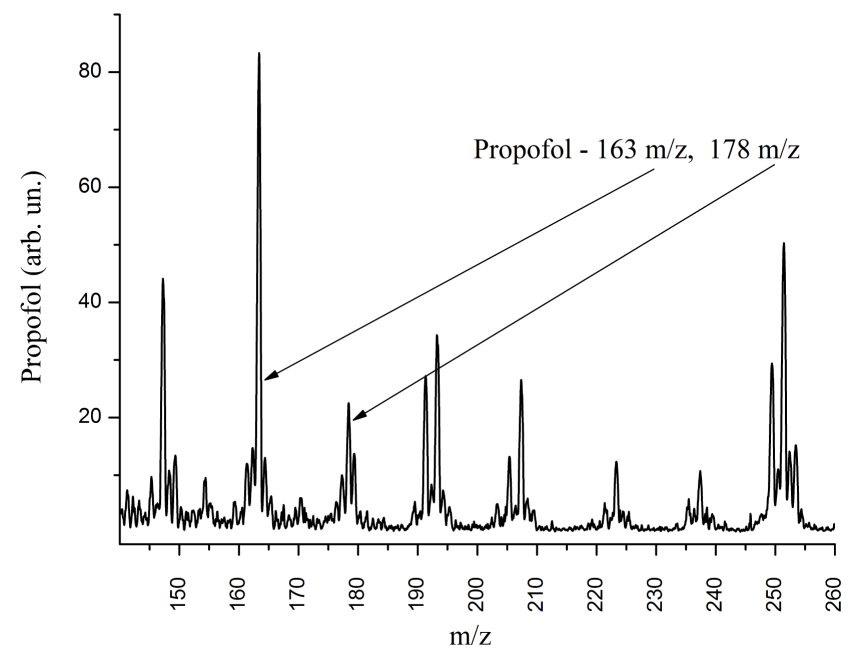

Figure 3. Mass-spectrum of fentanyl in the plasma that was taken during balanced inhalational anaesthesia, obtained using the membrane separator interface.

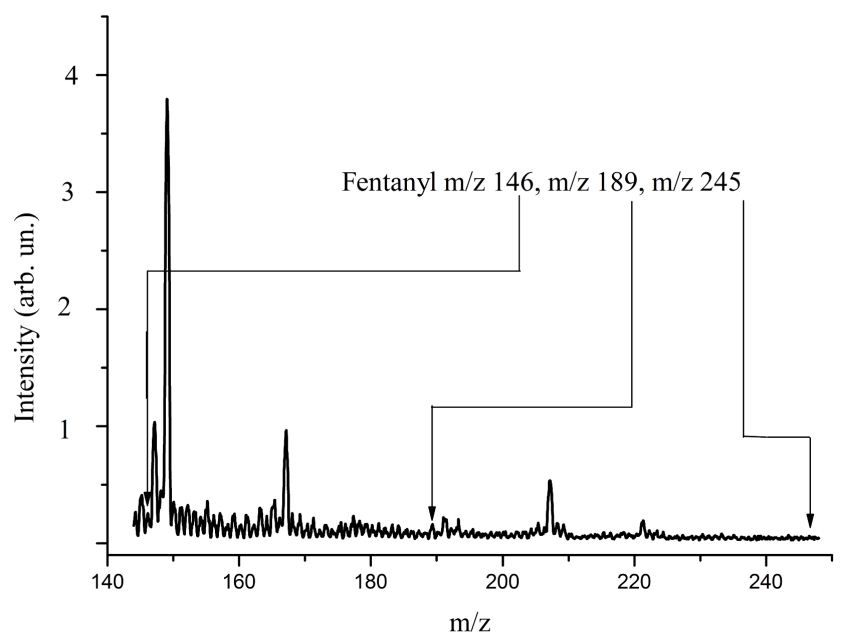

Figure 4. Mass-spectra of propofol in the plasma that was taken during total intravenous propofol-fentanyl anaesthesia, obtained using the membrane separator interface. 
propofol solution in the Intralipid drug for parenteral (intravenous) nutrition (Fresenius-Kabi) was prepared to serve as an internal standard; its composition is consistent with that of the propofol dissolution medium (Fresenius-Kabi). Based on four measurements of propofol concentration in plasma, it was equal to the specified target concentration of propofol in the program "Stanpump" with accuracy of at least 5.0\%. High solubility of propofol in the lipids allows its easy entrance to the CNS, which explains its rapid action. In the present study, blood samples were taken from PV and CSAB. Propofol concentration measurements in plasma for these two samples were performed using the membrane separator interface. The propofol concentration in the chiasmosellar area was Plasma $(\mathrm{PV}) /$ Plasma $(\mathrm{CSAB})=1.45 \pm 0.2$ less than in peripheral vein (see Figure 2). Estimation of the ratios for propofol concentrations was performed using fragmentation mass peaks of propofol $163 \mathrm{M} / \mathrm{Z}$ (see Figure 4).

\section{Conclusion}

As a result of the performed measurements (up to 30 cycles) no degradation of membrane properties was found. Duration of one measurement is $1 \mathrm{~min}$. Membrane interface is easy to use and maintain and has a potential for use in practical anaesthesiology for express analysis of anaesthetic drug concentration in plasma and CSF. The disadvantages of membrane interfaces include a relatively large response time in comparison to that of capillary input, dependence of the embrane characteristics on the temperature, and differential selectivity with respect to various compounds.

\section{Acknowledgements}

The authors thank the staff of the department of anesthesiology and critical medical care and the clinic of neurosurgery at the Kirov Medical Academy for assistance.

\section{References}

[1] Douglas, E.J., Plummer, G.F. and Cosgrove, M.B. (1981) Estimation of ICI 35,868 (Diprivan) in Blood by High-Performance Liquid Chromatography. Journal of Chromatography B: Biomedical Sciences and Applications, 223, 232237. http://dx.doi.org/10.1016/S0378-4347(00)80092-4

[2] Rohrig, T.P. and Moore, C. (2003) The Determination of Morphine in Urine and Oral Fluid Following Ingestion of Poppy Seeds. Journal of Analytical Toxicology, 27, 449-452. http://dx.doi.org/10.1093/jat/27.7.449

[3] Yu, H.Y. and Liau, J.K. (1993) Quantitation of Propofol in Plasma by Capillary Gas Chromatography. Journal of Chromatography B: Biomedical Sciences and Applications, 615, 77-81. http://dx.doi.org/10.1016/0378-4347(93)80292-C

[4] Johnson, R.C., Cooks, R.G., Allen, T.M., Ciser, M.E. and Hemberger, P.H. (2000) Membrane Introduction Mass Spectrometry: Trends and Applications. Mass Spectrometry Reviews, 19, 1-37. http://dx.doi.org/10.1002/(SICI)1098-2787(2000)19:1<1::AID-MAS1>3.0.CO;2-Y

[5] Rubin, L.L., Barbu, K., Bard, F., Cannon, C., Hall, D.E., Horner, H., Janatpour, M., Liaw, C., Manning, K., Morales, J., Porter, S., Tanner, L., Tomaselli, K. and Yednock, T. (1992) A Cell-Culture Model of the Blood-Brain-Barrier. New York Academy of Sciences, 663, 420-425.

[6] Yasuda, N., Targ, A.G. and Eger, E.I. (1989) Solubility of 1-653, Sevoflurane, Isoflurane, and Halothane in Human Tissues. Anesthesia \& Analgesia, 69, 37-43.

[7] Rosales, C.M., Young, T., Laster, M.J., Eger, E.I. and Garg, U. (2007) Sevoflurane Concentrations in Blood, Brain, and Lung after Sevoflurane-Induced Death. Journal of Forensic Sciences, 52, 1408-1417. http://dx.doi.org/10.1111/j.1556-4029.2007.00556.x

[8] Kaznessis, Y.N. (2005) A Review of Methods for Computational Prediction of Blood-Brain Partitioning. Curr. Med. Chem. Central Nervous System Agents, 5, 1-7. http://dx.doi.org/10.2174/1568015054863864

[9] Henthorn, T.K., Liu, Y., Mahapatro, M. and Ka-Yun, N.G. (1999 Active Transport of Fentanyl by the Blood-Brain Barrier. Journal of Pharmacology and Experimental Therapeutics, 289, 1084-1089.

[10] Shafer, S.L. (1996) STANPUMP User's Manual. Stanford University, Stanford. 